\title{
Left-Right Asymmetry of Maturation Rates in Human Embryonic Neural Development
}

\author{
Carolien G.F. de Kovel, Steven Lisgo, Guy Karlebach, Jia Ju, Gang Cheng, Simon E. Fisher, \\ and Clyde Francks
}

\begin{abstract}
BACKGROUND: Left-right asymmetry is a fundamental organizing feature of the human brain, and neuropsychiatric disorders such as schizophrenia sometimes involve alterations of brain asymmetry. As early as 8 weeks postconception, the majority of human fetuses move their right arms more than their left arms, but because nerve fiber tracts are still descending from the forebrain at this stage, spinal-muscular asymmetries are likely to play an important developmental role.

METHODS: We used RNA sequencing to measure gene expression levels in the left and right spinal cords, and the left and right hindbrains, of 18 postmortem human embryos aged 4 to 8 weeks postconception. Genes showing embryonic lateralization were tested for an enrichment of signals in genome-wide association data for schizophrenia. RESULTS: The left side of the embryonic spinal cord was found to mature faster than the right side. Both sides transitioned from transcriptional profiles associated with cell division and proliferation at earlier stages to neuronal differentiation and function at later stages, but the two sides were not in synchrony $(p=2.2 \mathrm{E}-161)$. The hindbrain showed a left-right mirrored pattern compared with the spinal cord, consistent with the well-known crossing over of function between these two structures. Genes that showed lateralization in the embryonic spinal cord were enriched for association signals with schizophrenia $(p=4.3 \mathrm{E}-05)$.

CONCLUSIONS: These are the earliest stage left-right differences of human neural development ever reported. Disruption of the lateralized developmental program may play a role in the genetic susceptibility to schizophrenia.
\end{abstract}

Keywords: Development, Gene expression, Hindbrain, Lateralization, Maturation rate, Schizophrenia, Spinal cord

http://dx.doi.org/10.1016/j.biopsych.2017.01.016

Many human cognitive processes are partially lateralized toward either the left or right side of the brain (1). Identifying genes involved in brain asymmetry is important because it can shed light on developmental processes that fine-tune neural circuits for their high-level functions $(2,3)$ and because various psychiatric and cognitive disorders can involve alterations of brain asymmetries $(4,5)$. At 8 weeks postconception, or roughly embryonic Carnegie stage (CS) 23, $85 \%$ of fetuses move their right arms more than their left arms, a proportion strikingly similar to the adult rate of right handedness (6). Furthermore, fetal hand motor asymmetry at 13 weeks postconception predicts handedness at age 12 years (7). Left-right anatomical differences of the cerebral hemispheres have been detected by ultrasound scanning as early as 18 weeks postconception (8). In infants, the right hemisphere has been reported to develop functionally and anatomically earlier than the left hemisphere in various respects, including that left hemisphere language-related brain regions develop more slowly than their contralateral counterparts (9-11). These observations indicate a broadly lateralized program of motor and cognitive development that begins in utero in our species and suggest that a general model can involve different rates of maturation on the two sides of the central nervous system (CNS).
Gene expression profiling from the left and right cerebral cortices in postmortem human fetuses has revealed little, or only tentative, evidence for laterality of messenger RNA (mRNA) expression in utero (12-15). One study, however, suggested that in 12- to 17-week-old fetuses the right cerebral cortex matures faster than the left one in terms of gene expression profiles (16). However, the corticospinal tract, which descends from the motor and somatosensory cerebral cortices to the spinal cord, only reaches the point of left-right crossover in the inferior hindbrain at 8 weeks postconception -the age of observed lateralization of arm movements-but does not yet extend into the spinal cord (17). Thus, lateralization of motor behavior at this stage is unlikely to reflect topdown asymmetry projected from the cerebral cortex. Instead, a bottom-up model is that molecular asymmetries already arise in the developing spinal cord prior to 8 weeks postconception. Developing vertebrate spinal cords show spontaneous electrophysiological activity that is affected by the formation of chemical synaptic networks (18), such that any left-right molecular differences might be reflected in patterns of spontaneous limb movements. In this study, we used RNA sequencing to measure gene expression profiles in the left and right spinal cords and hindbrains of human embryos aged 
4 to 8 weeks postconception to gain new insights into leftright differentiation of the human CNS within the earliest period of development yet analyzed in this way.

People with schizophrenia have an elevated rate of left handedness (19) and have shown alterations of various aspects of brain asymmetry in some, but not all, studies (20-26). Recent genome-wide association analysis in tens of thousands of participants has implicated individual genes and genetic networks involved in embryonic and fetal brain development in susceptibility to schizophrenia, bipolar disorder, and depression (27-29). Studies of patient-derived neural progenitor cells have also indicated disruptions of early developmental processes in schizophrenia (30). Therefore, we hypothesized that genes showing early developmental lateralization in the spinal cord or hindbrain might also show an enrichment of association signals within genome-wide association study (GWAS) results for schizophrenia. Although spinal cord abnormalities are not a known feature of schizophrenia, if subtle embryonic asymmetries within the spinal cord are important for patterning broader asymmetries of the brain later in development, then it is possible that disruptions of this early program might have consequences for disorder susceptibility.

\section{METHODS AND MATERIALS}

\section{Differential Expression Analysis}

RNA sequencing was performed separately for left and right spinal cords, and for left and right hindbrains, dissected from 18 postmortem human embryos aged 4 to 8 weeks postconception at the times of their terminations (CS13 to CS23; Supplemental Table S1), which had been donated to the United Kingdom's Human Developmental Biology Resource following voluntary medical abortions. Details of sample collection, dissection, preparation, RNA sequencing, and data quality control can be found in the Supplement. In RStudio (version 99.9.9; Boston, MA), gene mRNA expression data were normalized and transformed into log2 (counts per million [cpm]). Genes were filtered to retain only those for which at least three libraries had at least five reads per gene, separately for each brain structure. Linear models with observationallevel weights were fitted to obtain average expression values for each gene on each side (left or right), separately for spinal cord and hindbrain, and moderated $t$ statistics were used to assess differential expression between sides using the Bioconductor limma package (version 3.22.7; Bioconductor, Roswell Park Cancer Institute, Buffalo, NY; limma package, Walter \& Eliza Hall Institute for Medical Research, Melbourne, Australia) with voom (31). This design allowed paired analysis of the left and right samples from the same embryo for each tissue. In multidimensional scaling analysis of the gene expression data, no dimensions were found to relate to collection batch or time from clinic until freezing, and the number of pairs of samples was relatively low to support the inclusion of covariate effects (beyond the individual-level effect that was already accounted for by paired-sample testing). Therefore, we did not consider these factors in the differential expression analysis.

Many genes showed a pattern of increasing or decreasing expression with age, as assessed across the different embryos of varying ages. A linear slope was fitted through bilateral expression as log2 (cpm) on CS for each gene. The slope coefficient of each gene was plotted against the left-right differential expression $t$ value, and the Pearson correlation between the slope coefficients and $t$ values was computed (SPSS version 20.0.0.1; IBM Corp., Armonk, NY).

\section{Gene Ontology Enrichment}

Gene Ontology (GO) assigns genes to approximately 4000 gene sets representing biological processes in a loosely hierarchical structure (32). To search for biological process gene sets that were enriched on the left or right side of each structure, we used the Gene Set Enrichment Analysis (GSEA) tool from the Broad Institute (build 00044, January 2016; Cambridge, MA) combined with Molecular Signatures Database gene sets (c5.bp.5.1; http://www.go2msig.org/cgi-bin/ prebuilt.cgi), curated genes updated April 2015 (33,34). Analysis was performed using the preranked mode provided in the software. For this analysis, genes were ranked by lateralization $t$ value from positive (higher expression on the right) to negative (higher expression on the left) $t$ values. This way, positive enrichment in the GSEA results would indicate that members of a particular gene set, on average, show relatively higher expression on the right side of the tissue, and negative enrichment would indicate relatively higher expression on the left side. GSEA reports a familywise error rate, which is a conservative measure to correct for multiple testing (35). To identify gene sets that were lateralized in both spinal cord and hindbrain, we selected gene sets that had an absolute normalized enrichment score (as calculated by GSEA software) exceeding 2.0 in both tissues. To cluster the GO gene sets into larger units in order to indicate the broad types of processes implicated, REVIGO (http://revigo.irb.hr/) was used (36). See Supplement for more details.

\section{Transcription Factor Target Enrichment}

To identify transcription factors (TFs) that play important roles in lateralization of spinal cord and hindbrain, we performed enrichment analysis for TF targets in our left-right differential expression data. Gene sets, provided by Molecular Signatures Database, were characterized by having a particular target motif near their transcription start site. Gene set collection c3.tft.5.0 from Molecular Signatures Database, updated June 2015, contained 615 gene sets. Enrichment analysis was performed as described for GO gene sets above. See Supplement for more details.

\section{Overlap With Schizophrenia GWAS Results}

We downloaded the publicly available association statistics from the Psychiatric Genomics Consortium schizophrenia genome-wide association study of up to 36,989 cases and 113,075 controls (https://www.med.unc.edu/pgc/results-anddownloads; SCZ2) (29). We tested the hypothesis that genes that showed lateralized expression in the embryonic spinal cord or hindbrain were enriched for association signals with schizophrenia by running the PASCAL software (http://www2. unil.ch/cbg/index.php?title=Pascal) (35). For this, we defined one gene set comprising the 681 genes that showed lateralization (toward either the right or the left) in the embryonic 


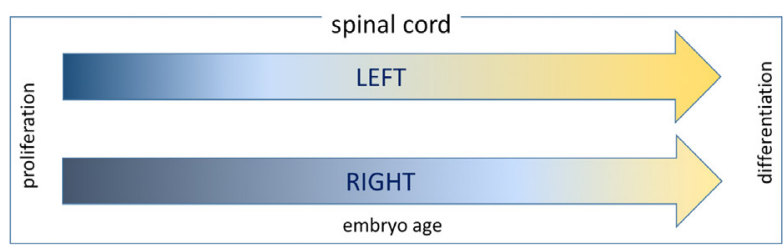

A

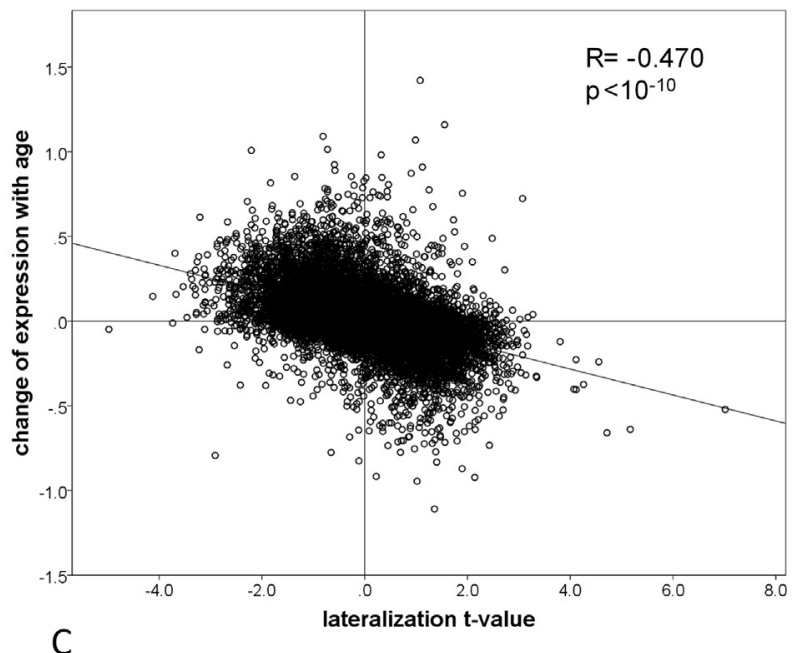

C

SHANK1 expression

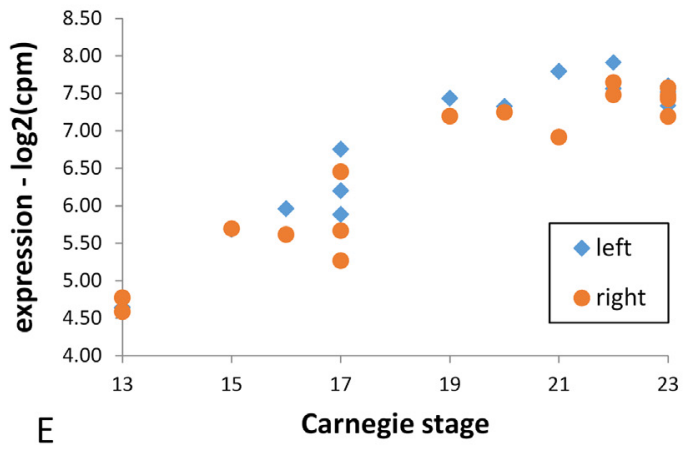

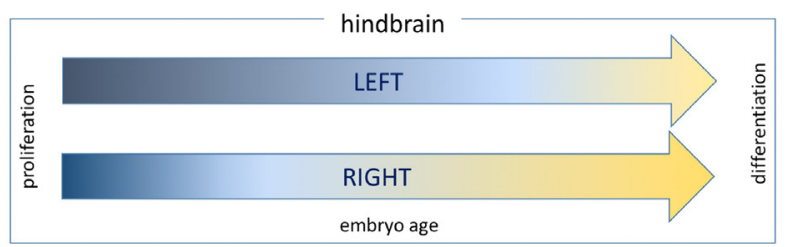

B

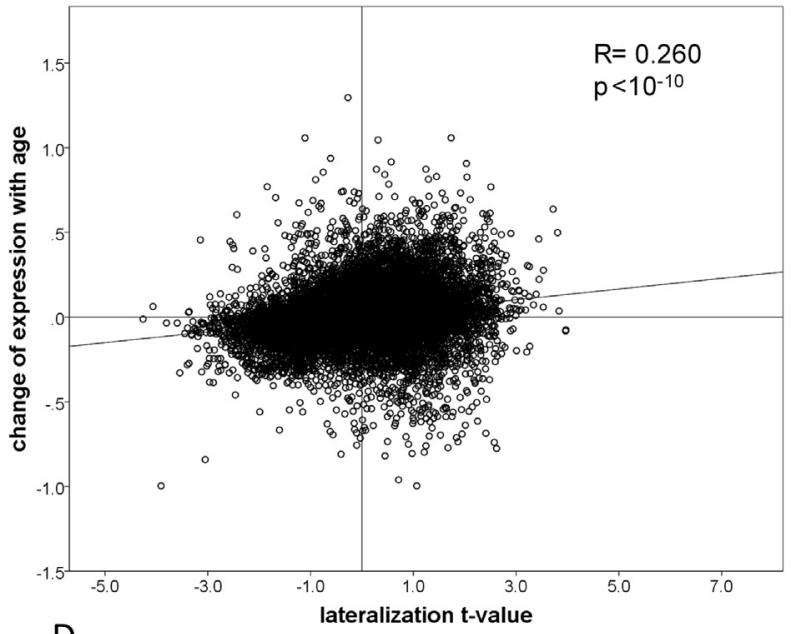

D

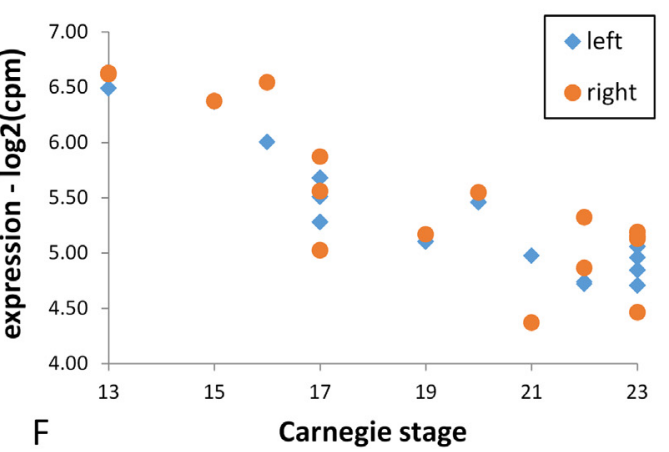

Figure 1. Developmental changes in gene expression from CS13 to CS23. (A, B) Schematic figures to indicate left-right differential rates of maturation in the spinal cord (A) and hindbrain (B). Both sides were found to transition from transcriptional profiles of earlier proliferation to later neuronal differentiation, but not synchronously with each other. (C, D) The relation of per-gene bilateral changes in gene expression ( $y$ axis: slope of bilateral change) to differential leftright expression ( $x$ axis: lateralization $t$ value) within the spinal cord (C) and hindbrain (D). R, Pearson's correlation; $p, p$ value for correlation. In the spinal cord, genes showing higher expression on the left side (negative lateralization $t$ ) also tend to increase in expression with age (positive bilateral change), whereas those with lower expression in the left spinal cord also tend to decrease with age. In the hindbrain, the patterns are left-right mirrored compared with the spinal cord. (E, F) Illustrative examples of individual genes showing large changes of expression levels from CS13 to CS23 in the spinal cord. CS, Carnegie stage.

spinal cord with an absolute $t$ value $>2.0$ for the left-right comparison and defined another gene set for the 816 genes that showed lateralization with an absolute $t$ value $>2.0$ in the embryonic hindbrain. Further gene sets, also defined from the transcriptomic data, were tested to better understand the factors driving the results obtained with the first two sets (see Results). Briefly, PASCAL uses per-single nucleotide polymorphism association statistics, as derived from a GWAS study, to compute one association score for every known gene. Information on linkage disequilibrium between single nucleotide polymorphisms is included in this step. The score for a given gene therefore indicates how strongly genetic variation within, or in linkage disequilibrium with, that gene is associated with schizophrenia. These GWAS-based gene scores are subsequently used to compute gene set enrichment within defined sets of genes (here as defined from the transcriptomic data). The enrichment analysis tests whether the genes in a given set have, on average, higher GWASbased gene scores than the other genes in the genome. No cutoff was made on the GWAS-based gene scores, so that for 
A

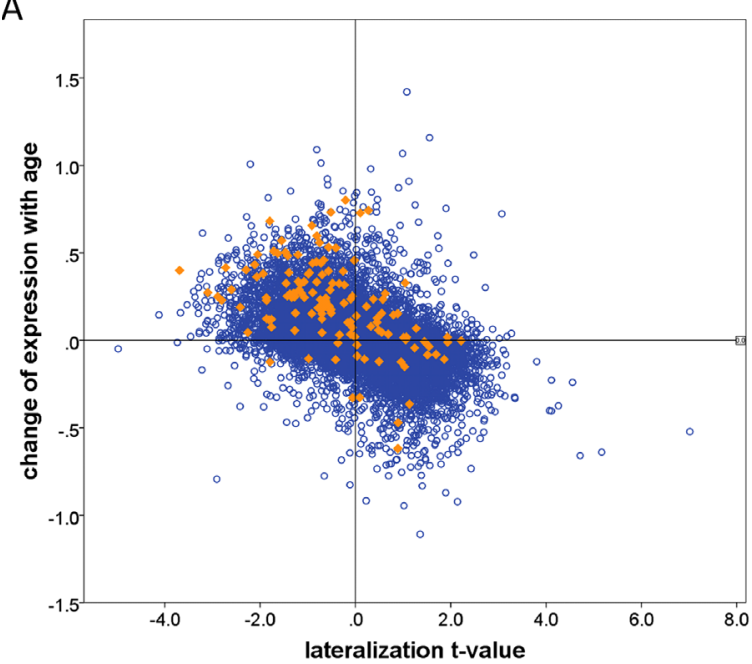

C

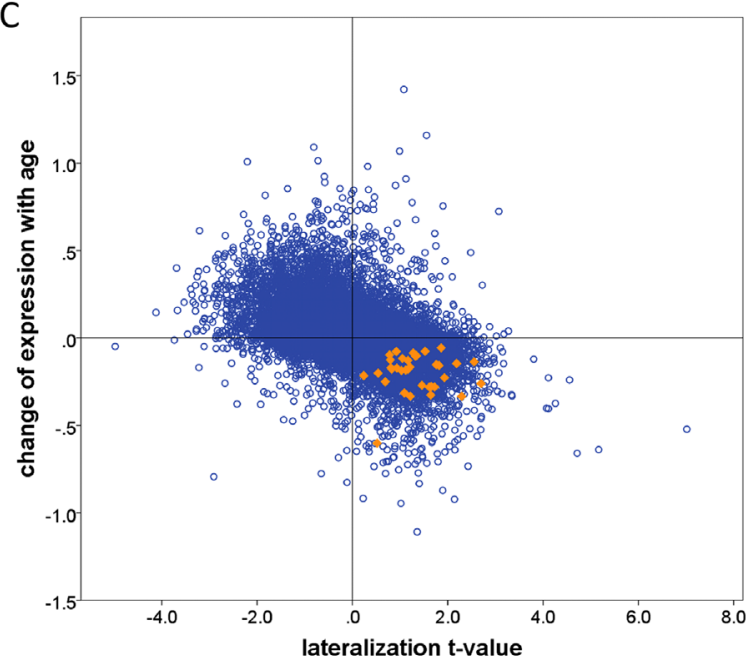

B
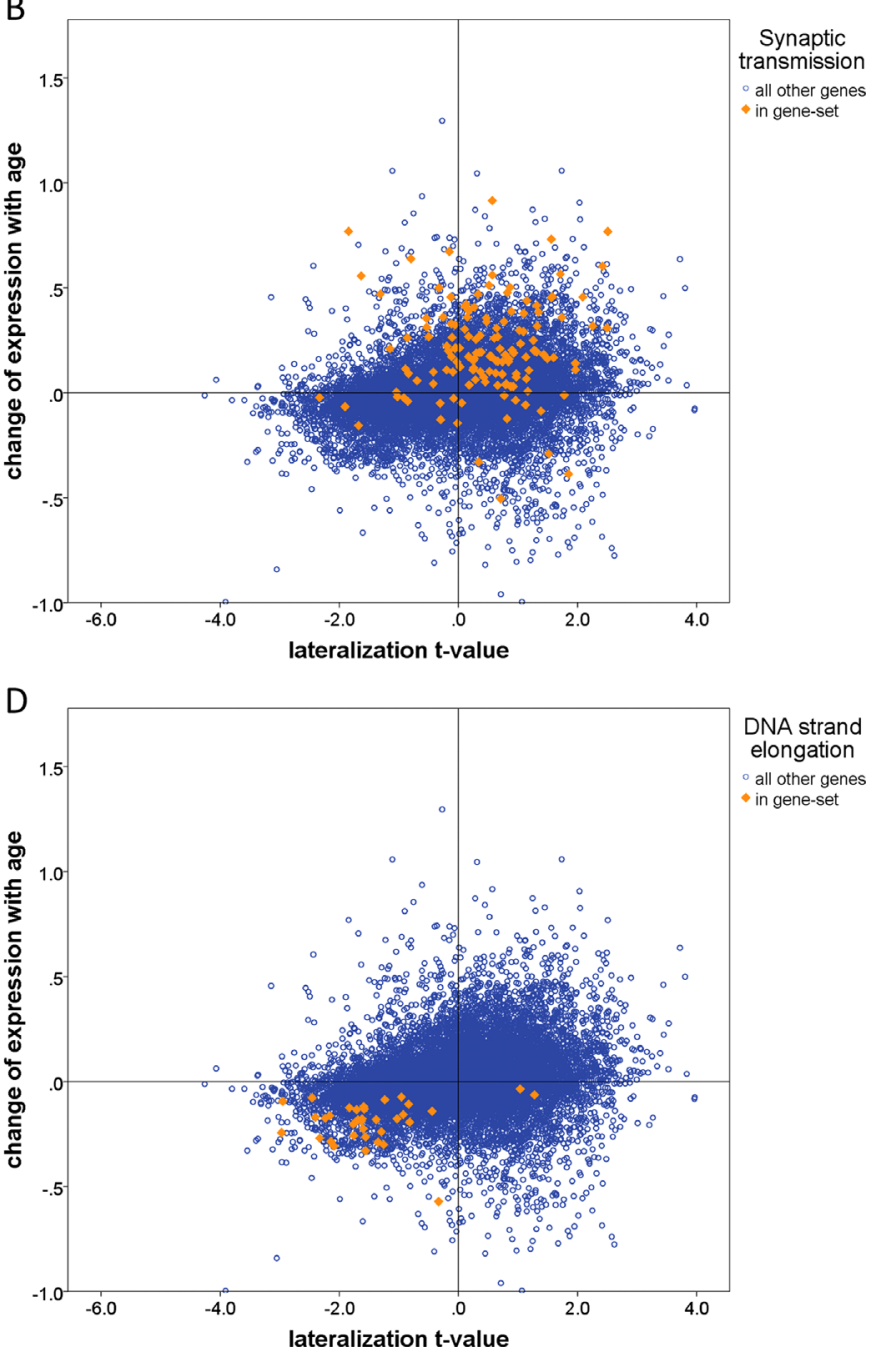

Figure 2. Examples of Gene Ontology (GO) gene set enrichment mirrored between the spinal cord and hindbrain. $x$ axis: left-right differential expression $t$ value; $y$ axis: regression slope of expression (as log2 [counts per million]) on embryonic age (Carnegie stage); orange: genes within the gene set; blue: all other genes. (A, B) Genes in gene set "synaptic transmission" (GO:0007268) increase in expression with age and are more highly expressed in the left spinal cord (normalized expression score $[N E S]=2.65$, familywise error rate $[F W E R]<5.0$ E-04) $(\mathbf{A})$ and the right hindbrain $(\mathrm{NES}=2.15, \mathrm{FWER}=0.034)(\mathbf{B})$. (C, D) Genes in gene set "DNA strand elongation" (GO:0022616) decrease in expression with age and are expressed at a lower level in the left spinal cord (NES = 2.41, FWER $<5.0$ E-04) (C) and the left hindbrain (NES = 2.68, FWER $<5.0$ E-04) (D).

all genes the degree of association with schizophrenia was taken into account. We used the option "max" in PASCAL for evaluating the gene scores, combined with the rank-based option "chi" for evaluating gene set enrichment, and otherwise default settings.

\section{Data Accessibility}

RNA sequencing data have been deposited in the Gene Expression Omnibus under accession number GSE84231.

\section{RESULTS}

\section{Asymmetrical Gene Expression}

We carried out transcriptomic analysis by RNA sequencing of the left and right spinal cords, and the left and right hindbrains, of 18 normally developing human embryos aged 4 to 8 weeks postconception at the times of their terminations (CS13 to CS23), which had been voluntarily donated by women after they chose to end their pregnancies (Supplemental Table S1). The sequenced libraries had a median number of 47.6 E06 $(\mathrm{SD}=11.9$ E06) clean reads. The numbers of genes included in the analyses after filtering were 13,852 genes in the spinal cord and 13,730 genes in the hindbrain. Average gene expression as log2 (cpm) was $4.86(\mathrm{SD}=2.05)$ for the spinal cord and 4.87 (SD $=2.04$ ) for the hindbrain. In multidimensional scaling analysis of the transcriptomic data (Supplemental Figure S1), the first dimension reflected embryonic age, the second dimension reflected the hindbrain-spinal cord distinction, and the paired left-right samples from a given embryo mapped closely together.

Left-right differential expression per gene was then assessed, separately within each structure, using a paired sample design and by combining data from all embryos. 
Overall, lateralization of gene expression was subtle, with no individual genes showing significant left-right differences in expression levels at a false discovery rate $<0.01$ after correcting over all genes tested. Multidimensional scaling analysis separately within the spinal cord and hindbrain also did not identify any of the top 10 dimensions as being associated with the left-right distinction (not shown). Many genes showed dramatic bilateral decreases in spinal cord expression from CS13 to CS23, while other genes showed strong bilateral increases in expression over this period (see Figure 1E, F for illustrative examples). We assigned every gene a linear slope for its bilateral expression change in the spinal cord from CS13 to CS23 and found a correlation ( $r=-.471$, $p=2.2 \mathrm{E}-161)$ between this slope and the left-right lateralization of genes (Figure 1C). Genes whose spinal cord expression levels decreased bilaterally from CS13 to CS23 also tended to show lower expression on the left side, while genes whose spinal cord expression levels increased bilaterally from CS13 to CS23 tended to show higher expression on the left side. Therefore, the global developmental changes in the transcriptome over the embryonic period CS13 to CS23, which both sides of the spinal cord followed, were at an advanced state in the left spinal cord compared with the right spinal cord (Figure 1A).

In GO analysis of spinal cord data, the left side showed, relative to the right side, higher expression of genes involved in glutamate receptor signaling and neurotransmitter transport (Figure 2, Supplemental Figure S2A, and Supplemental Table S2), which also tended to be genes whose average expression increased bilaterally from $\mathrm{CS} 13$ to $\mathrm{CS} 23$, consistent with an increase in neuronal differentiation and functional maturation over this period, which was advanced on the left. In contrast, the left spinal cord showed lower expression than the right spinal cord for genes involved in diverse intracellular processes, including mRNA metabolism, DNA strand elongation, chromosome segregation, and protein translation (Figure 2, Supplemental Figure S2B, and Supplemental Table S2), and these gene sets decreased bilaterally from CS13 to CS23, such that again the left side was relatively advanced. These transcriptional profiles indicate a rightside delay, in the spinal cord, of phasing out cellular division/ proliferation processes and transitioning to neuronal differentiation and functional maturation (Figure 1A). Further quantification of the left-right asynchrony, with respect to the most lateralized gene sets, can be found in Supplemental Figure S4.

The hindbrain showed essentially a left-right mirrored, but weaker, pattern compared with the spinal cord, regarding the relation of lateralization to bilateral changes in gene expression levels from CS13 to CS23 (Figures 1-3, Supplemental Figure S2C, D, Supplemental Figure S3, and Supplemental Tables S3 and S5). The correlation between lateralization and bilateral developmental change in expression, as seen in panels $C$ and D of Figure 1, was significantly weaker in the hindbrain than in the spinal cord (Fisher $r$-to- $Z$ transformation to compare correlations, $Z=20.23, p=5.33 \mathrm{E}-91)$. Per-gene comparisons of hindbrain and spinal cord laterality and developmental changes in expression can be found in Supplemental Figures S5 and S6.

\section{TF Target Genes}

TFs are proteins that regulate the expression levels of their target genes through binding to specific DNA sequence motifs that are located in the vicinity of their target genes in the genome $(9,11)$. We used binding motif data for each of 615 motifs to define sets of TF target genes. We found significant enrichment, after multiple testing correction, for targets of the TF NRSF (neuron-restrictive silencer factor; coded by the gene $R E S T$ ) among genes showing leftward lateralization in the spinal cord, with a mirror effect in the hindbrain (Figure $3 \mathrm{~A}, \mathrm{~B}$ ). In addition, targets for SF1 and TCF3 were significantly enriched among left-lateralized genes. Similarly, targets of the TF E2F1 (E2F transcription factor 1) were significantly enriched among genes showing rightward lateralization in the spinal cord (and leftward lateralization in the hindbrain) (Figure 3C, D) as well as targets of TFDP1 (Supplemental Tables S4 and S5). NRSF is a repressor of neuronal gene transcription in non-neuronal cells $(37,38)$. Consistent with this role, its own mRNA lateralization was contralateral to that of its target set, and it decreased bilaterally in expression from CS13 to CS23 while its targets increased (Figure 1 and Figure 3A, B). In contrast, E2F1, which had targets expressed more highly on the right, is one of the most important proteins for entry into the $\mathrm{S}$ phase and progression of the cell cycle and is a promoter of proliferation $(39,40)$. Again our data matched the known biology; E2F1 lateralization was ipsilateral to its targets, whose expression decreased bilaterally in concert with it from CS13 to CS23 (Figure 3C, D). Thus, we identified individual TFs that are likely to exert developmental effects on lateralized maturation rates in the spinal cord and hindbrain, again with respect to transitioning from proliferation to neuronal differentiation, in an analysis that was independent of GO analysis. More details can be found in the Supplement.

\section{Association With Schizophrenia}

Within the Psychiatric Genomics Consortium schizophrenia phase 2 genome-wide association results, genes that were lateralized in the embryonic spinal cord with an absolute $t$ value $>2.0$ (i.e., the most strongly left and right lateralized genes) were significantly enriched for single nucleotide polymorphisms showing association with schizophrenia $(p=4.3$ E-05), while no such signal was seen for genes that were lateralized with an absolute $t$ value $>2.0$ in the embryonic hindbrain $(p=.087)$. We further tested a set of 758 genes whose developmental change in expression was $>0.3(\log 2$ [cpm]/Carnegie stage) (i.e., those genes whose expression increased most strongly from CS13 to CS23), which also tended to be more highly expressed on the left (Figure 1C), and again we found an enrichment of association signals with schizophrenia ( $p=3.3 \mathrm{E}-06$ ). No such enrichment was found $(p=.994)$ among the 647 genes that decreased most strongly in their expression bilaterally from CS13 to CS23 (i.e., genes with log2 [cpm]/Carnegie stage $<-0.3$ ), and that also tended to be expressed at lower levels on the left (Figure 1C). This indicates that the enrichment of association signals with schizophrenia was largely driven by genes of mature neuronal function that strongly increase bilaterally in expression during nervous system development and also tend to be more highly expressed in the left embryonic spinal cord. However, we also tested the set of 149 left-lateralized genes $(t<-2.0)$, which showed log2 (cpm)/Carnegie stage $<0.2$ (i.e., those with only weakly increasing, or else decreasing, 
A

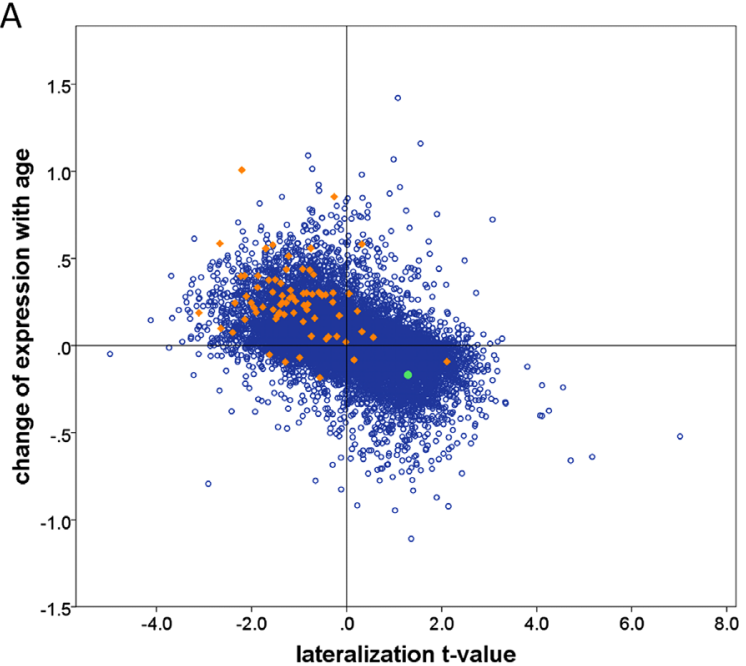

C

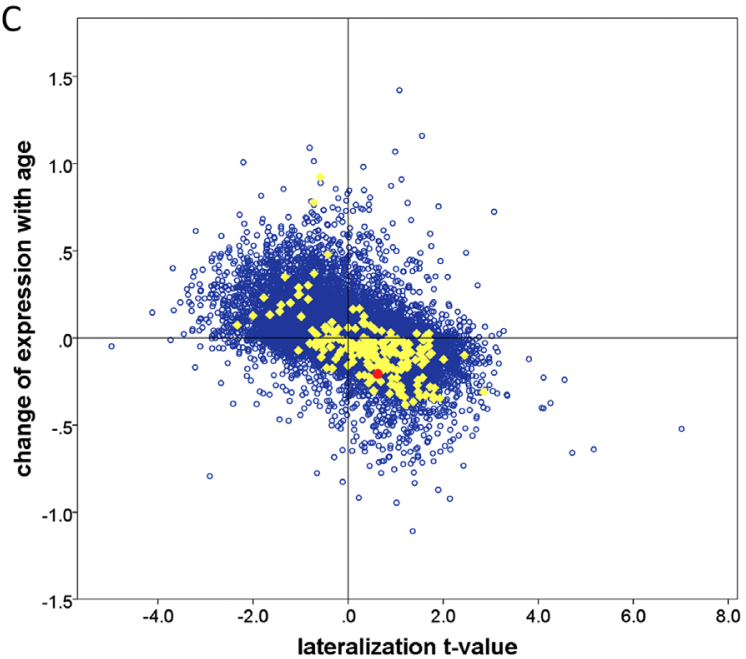

B
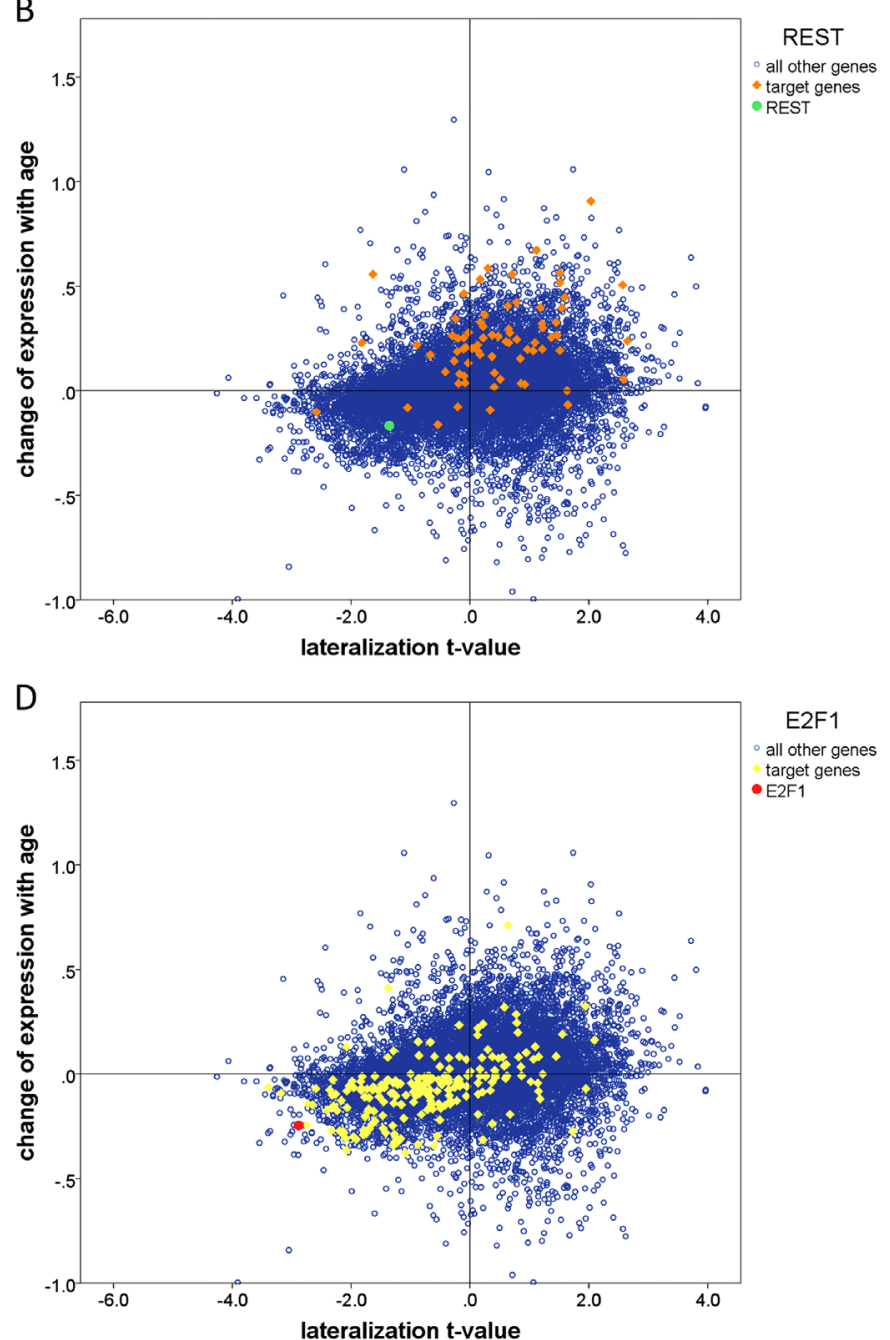

Figure 3. Lateralization of transcription factor (TF) target gene sets. (A, B) Targets of the TF neuron-restrictive silencer factor (NRSF) in the spinal cord (A) and hindbrain (B). $y$ axis: slope of change in bilateral expression with embryonic age; $x$ axis: left-right differential expression $t$ value; orange: genes with a target motif of NRSF; green: NRSF itself; blue: all other genes in the dataset. Genes that have the motif, in cis, that is targeted by the repressor NRSF are more highly expressed in the left spinal cord (normalized expression score [NES] $=3.14$, familywise error rate [FWER] $<5.0 \mathrm{E}-04$ ) and increase in expression with age. The pattern is left-right mirrored in the hindbrain. (C, D) Targets of the TF E2F transcription factor 1 (E2F1) in the spinal cord (C) and hindbrain (D). Axes and labels are as for (A, B) except that E2F1 itself is shown in red and its targets are shown in yellow. Genes that have the motif TTTSCGS in cis, which is targeted by E2F1, are more weakly expressed in the right hindbrain (NES $=2.66$, FWER $<5.0 \mathrm{E}-04$ ) and decrease in expression with age. This lateralization is left-right mirrored in the spinal cord.

bilateral expression levels from CS13 to CS23), and again found an enrichment of association signals with schizophrenia $(p=.017)$, so that it was not only genes showing a marked expression increase with developmental age that are associated with schizophrenia.

\section{DISCUSSION}

In this study, we investigated whether the human embryonic spinal cord and hindbrain would show left-right transcriptional differences, consistent with a bottom-up model in which inferior regions of the CNS may lead its lateralized development. This was motivated by observations from in utero ultrasound scanning, which have indicated that asymmetries of motor behavior precede the formation of the corticospinal tract (41) and therefore possible top-down influences from the cerebral cortex.

We found that, from 4 to 8 weeks postconception (i.e., CS13 to CS23), both sides of the embryonic spinal cord transition from transcriptional profiles associated with cell division and proliferation at earlier stages to neuronal differentiation and function at later stages, but the two sides are not in synchrony with each other. The left spinal cord follows a slightly accelerated developmental trajectory compared with the right spinal cord. The hindbrain showed a left-right mirrored, but weaker, pattern compared with the spinal cord. These are the earliest left-right differences in human neural development to have ever been reported. 
The transcriptional mirroring between the spinal cord and hindbrain is broadly consistent with the well-established crossing over (decussation) of structure and function in the inferior hindbrain (42). Because the corticospinal tract only arrives at the inferior hindbrain by CS23 (17), any molecular asymmetry in descending axons would not be expected to affect the spinal cord directly, although it may affect the hindbrain by CS23. Therefore, in the spinal cord particularly, transcriptional asymmetry from CS13 to CS23 is likely to originate independently of top-down forebrain influences. Studies earlier than CS13 could pinpoint the developmental triggers for this lateralized program, although such samples are not usually available through voluntary termination of pregnancy. Upstream developmental triggers might occur within the precursors of the spinal cord itself, or within peripheral neural or even non-neural tissue, and may involve both genetic and environmental stimuli such as have been found in fish and bird species $(43,44)$.

The early lateralization in human spinal cord and hindbrain that we have identified may influence later asymmetries of the cerebral cortex (16), possibly via the formation of asymmetrical spinal-cortical circuitry and feedback. The slower transcriptional maturation that we observed of right spinal cord and left hindbrain is consistent with previously reported left hemisphere delayed development of the infant forebrain (17) and fetal cerebral cortex (16), at least with respect to the corticospinal tract and ascending proprioceptor and touch pathways, which cross over in the hindbrain but run ipsilaterally from forebrain to hindbrain. Therefore, our data support a general model in which left-right differences of maturational rates are fundamental to creating structural and functional lateralization of the human brain, as was discussed by Corballis and Morgan in 1978 (45), although those authors envisioned a generally left hemisphere maturational advantage. In terms of anatomy, the earliest developmental asymmetry of the human brain yet reported is a left $>$ right volumetric difference of the choroid plexuses at roughly 9 weeks postconception (46). In fact, the volume of a structure does not necessarily bear a direct relation to its maturation rate; for example, a prolonged developmental phase of cell proliferation prior to differentiation could result in a relatively larger structure, which would be due to a kind of early developmental delay. This consideration might help to reconcile some of the observations made by Corballis and Morgan (45).

We found that genes showing embryonic CNS lateralization in the spinal cord contain variants in the population that contribute to the polygenic liability to schizophrenia more often than the rest of the genes in the genome. Genes whose expression levels increased during the embryonic period, and also tended to be expressed slightly more strongly in the left spinal cord, played the largest role in this enrichment for schizophrenia association signals. These same genes tend to have mature neuronal functions, such as being involved in glutamatergic neurotransmission, and it is therefore possible that variants in these genes associate with schizophrenia due to later functions in the brain and not causally via alterations of an embryonic program for CNS laterality. However, we also found that a set of left-lateralized genes in the spinal cord that show relatively low developmental increases, and even decreases, also showed enrichment for association signals with schizophrenia, suggesting that laterality itself may be involved in disorder susceptibility. Given the strong relation between lateralization and developmental change of expression, with our data it is not possible to fully disentangle these aspects.

Lateralization of CNS structure and function is found across the vertebrates (47), probably reflecting the general dual advantages of efficiency of function within each of the two sides and division of labor between them. Some developmental mechanisms underlying human CNS lateralization are therefore likely to be evolutionarily ancient even if other aspects of the elaboration of asymmetry, such as for left hemisphere language in the typical human brain (42), are evolutionarily modern. In mice, different spinal interneuron populations derive from separate progenitor domains that have distinct transcriptional profiles, and the timing of neurogenesis also affects spinal cord neuronal diversification (42). Such high-resolution spatial and temporal analysis of human embryonic tissue is limited for practical and ethical reasons. Our data may point to a conserved set of asymmetry mechanisms that can be analyzed further in animals (47). However, the extent to which these mechanisms operate in different species could vary widely. The human populationlevel bias of roughly $90 \%$ toward right handedness is among the strongest known for any mammal, although this may relate to the human demands of very fine motor control and is likely to be reinforced and amplified as patterns of use diverge between the two limbs throughout development (48). Interestingly, while our data point to a lateralized developmental program that is under strong genetic control in typically lateralized humans, it should be noted that left handedness and other aspects of nontypical brain lateralization have been shown to be largely influenced by nongenetic factors $(49,50)$.

In summary, gene expression analysis of left and right spinal cord and hindbrain in human embryos revealed differences in transcriptional maturation rates. The asymmetrical patterns within the spinal cord and hindbrain were largely mirrored with respect to each other, which suggests that lateralized transcription is linked to descending and ascending neuronal tracts that cross over in the inferior hindbrain. Because descending tracts only arrive at the inferior hindbrain by CS23, embryonic lateralization within the spinal cord is not likely to be directed from the forebrain. Variants in genes that showed leftward lateralization in the embryonic CNS are involved in susceptibility to schizophrenia.

\section{ACKNOWLEDGMENTS AND DISCLOSURES}

CGFdK and GK were supported by an Open Programme grant (No. 824.14.005) to CF from the Netherlands Organisation for Scientific Research (NWO). CF and SEF are supported by the Max Planck Society (Germany). Human embryos were obtained from the Medical Research Council (MRC)/Wellcome Trust-funded Human Developmental Biology Resource (HDBR; http://www.hdbr.org; Grant No. 099175/Z/12/Z), with appropriate maternal written consent and approval from the Newcastle and North Tyneside National Health Service (NHS) Health Authority National Research Ethics Committee (REC; Reference No. 08/H0906/21+5). HDBR is licensed as a tissue bank by the U.K. Human Tissue Authority (HTA) and operates in accordance with all the relevant HTA codes of practice.

CF conceived the study and designed the experiments. CGFdK and GK analyzed the data. SL led the dissection of the samples. JJ and GC performed preliminary data analysis. SEF contributed to data interpretation. CF and CGFdK wrote the article. 
The authors report no biomedical financial interests or potential conflicts of interest.

\section{ARTICLE INFORMATION}

From the Language and Genetics Department (CGFdK, GK, SEF, CF), Max Planck Institute for Psycholinguistics, and Donders Institute for Brain, Cognition and Behaviour (SEF, CF), Radboud University Nijmegen, Nijmegen, The Netherlands; Institute of Genetic Medicine (SL), Newcastle, United Kingdom; and Beijing Genomics Institute-Shenzhen (JJ, GC), Shenzhen, China.

Address correspondence to Clyde Francks, D.Phil., Language and Genetics Department, Max Planck Institute for Psycholinguistics, Wundtlaan 1, 6525 XD Nijmegen, The Netherlands; E-mail: clyde.francks@mpi.nl.

Received Oct 11, 2016; revised Jan 20, 2017; accepted Jan 20, 2017.

Supplementary material cited in this article is available online at http:// dx.doi.org/10.1016/j.biopsych.2017.01.016.

\section{REFERENCES}

1. Toga AW, Thompson PM (2003): Mapping brain asymmetry. Nat Rev Neurosci 4:37-48.

2. Francks C (2015): Exploring human brain lateralization with molecular genetics and genomics. Ann N Y Acad Sci 1359:1-13.

3. Willems RM, der Haegen LV, Fisher SE, Francks C (2014): On the other hand: Including left-handers in cognitive neuroscience and neurogenetics. Nat Rev Neurosci 15:193-201.

4. Rentería ME (2012): Cerebral asymmetry: A quantitative, multifactorial, and plastic brain phenotype. Twin Res Hum Genet 15:401-413.

5. Okada N, Fukunaga M, Yamashita F, Koshiyama D, Yamamori H, Ohi $\mathrm{K}$, et al. (2016): Abnormal asymmetries in subcortical brain volume in schizophrenia. Mol Psychiatry 21:1460-1466.

6. Hepper PG, Shahidullah S, White R (1991): Handedness in the human fetus. Neuropsychologia 29:1107-1111.

7. Hepper PG, Wells DL, Lynch C (2005): Prenatal thumb sucking is related to postnatal handedness. Neuropsychologia 43:313-315.

8. Hering-Hanit R, Achiron R, Lipitz S, Achiron A (2001): Asymmetry of fetal cerebral hemispheres: In utero ultrasound study. Arch Dis Child 85:F194-F196.

9. Chiron C, Jambaque I, Nabbout R, Lounes R, Syrota A, Dulac O (1997): The right brain hemisphere is dominant in human infants. Brain 120:1057-1065.

10. Joyce JJ, Dickson PI, Qi N, Noble JE, Raj JU, Baylen BG (2004): Normal right and left ventricular mass development during early infancy. Am J Cardiol, 93, 797-801.

11. Leroy F, Glasel H, Dubois J, Hertz-Pannier L, Thirion B, Mangin JF, et al. (2011): Early maturation of the linguistic dorsal pathway in human infants. J Neurosci 31:1500-1506.

12. Johnson MB, Kawasawa YI, Mason CE, Krsnik Z, Coppola G, Bogdanovic D, et al. (2009): Functional and evolutionary insights into human brain development through global transcriptome analysis. Neuron 62:494-509.

13. Lambert N, Lambot M-A, Bilheu A, Albert V, Englert $\mathrm{Y}$, Libert F, et al. (2011): Genes expressed in specific areas of the human fetal cerebral cortex display distinct patterns of evolution. PLoS One 6:e17753.

14. Pletikos M, Sousa André MM, Sedmak G, Meyer KA, Zhu Y, Cheng F, et al. (2014): Temporal specification and bilaterality of human neocortical topographic gene expression. Neuron 81:321-332.

15. Abrahams BS, Tentler D, Perederiy JV, Oldham MC, Coppola G, Geschwind DH (2007): Genome-wide analyses of human perisylvian cerebral cortical patterning. Proc Natl Acad Sci U S A 104: $17849-17854$.

16. Sun T, Patoine C, Abu-Khalil A, Visvader J, Sum E, Cherry TJ, et al. (2005): Early asymmetry of gene transcription in embryonic human left and right cerebral cortex. Science 308:1794-1798.

17. ten Donkelaar HJ, Lammens M, Wesseling P, Hori A, Keyser A, Rotteveel J (2004): Development and malformations of the human pyramidal tract. J Neurol 251:1429-1442.
18. O'Donovan MJ (1999): The origin of spontaneous activity in developing networks of the vertebrate nervous system. Curr Opin Neurobiol 9:94-104.

19. Hirnstein M, Hugdahl K (2014): Excess of non-right-handedness in schizophrenia: Meta-analysis of gender effects and potential biases in handedness assessment. Br J Psychiatry 205:260-267.

20. Sun Y, Chen Y, Collinson SL, Bezerianos A, Sim K (2017): Reduced hemispheric asymmetry of brain anatomical networks is linked to schizophrenia: A connectome study. Cereb Cortex 27: $602-625$

21. Ocklenburg S, Güntürkün O, Hugdahl K, Hirnstein M (2015): Laterality and mental disorders in the postgenomic age-A closer look at schizophrenia and language lateralization. Neurosci Biobehav Rev 59:100-110.

22. Mueller S, Wang D, Pan R, Holt DJ, Liu H (2015): Abnormalities in hemispheric specialization of caudate nucleus connectivity in schizophrenia. JAMA Psychiatry 72:552-560.

23. Ribolsi M, Daskalakis ZJ, Siracusano A, Koch G (2014): Abnormal asymmetry of brain connectivity in schizophrenia. Front Hum Neurosci 8:1010.

24. Oertel-Knochel V, Knochel C, Stablein M, Linden DE (2012): Abnormal functional and structural asymmetry as biomarker for schizophrenia. Curr Top Med Chem 12:2434-2451.

25. Deep-Soboslay A, Hyde TM, Callicott JP, Lener MS, Verchinski BA, Apud JA, et al. (2010): Handedness, heritability, neurocognition and brain asymmetry in schizophrenia. Brain 133:3113-3122.

26. Atagun MI, Sikoglu EM, Soykan C, Serdar Suleyman C, UlusoyKaymak S, Caykoylu A, et al. (2017): Perisylvian GABA levels in schizophrenia and bipolar disorder. Neurosci Lett 637:70-74.

27. Network and Pathway Analysis Subgroup of Psychiatric Genomics Consortium (2015): Psychiatric genome-wide association study analyses implicate neuronal, immune and histone pathways. Nat Neurosci 18:199-209.

28. Birnbaum R, Jaffe AE, Chen Q, Hyde TM, Kleinman JE, Weinberger DR (2015): Investigation of the prenatal expression patterns of 108 schizophrenia-associated genetic loci. Biol Psychiatry 77:e43-e51.

29. Schizophrenia Working Group of the Psychiatric Genomics Consortium (2014): Biological insights from 108 schizophrenia-associated genetic loci. Nature 511:421-427.

30. Brennand K, Savas JN, Kim Y, Tran N, Simone A, Hashimoto-Torii K, et al. (2015): Phenotypic differences in hiPSC NPCs derived from patients with schizophrenia. Mol Psychiatry 20:361-368.

31. Law CW, Chen Y, Shi W, Smyth GK (2014): voom: Precision weights unlock linear model analysis tools for RNA-seq read counts. Genome Biol 15:R29.

32. Gene Ontology Consortium, Ashburner M, Ball CA, Blake JA, Botstein D, Butler H, et al. (2000): Gene ontology: Tool for the unification of biology. Nat Genet 25:25-29.

33. Mootha VK, Lindgren CM, Eriksson KF, Subramanian A, Sihag S, Lehar J, et al. (2003): PGC-1 $\alpha$-responsive genes involved in oxidative phosphorylation are coordinately downregulated in human diabetes. Nat Genet 34:267-273.

34. Subramanian A, Tamayo P, Mootha VK, Mukherjee S, Ebert BL, Gillette MA, et al. (2005): Gene set enrichment analysis: A knowledgebased approach for interpreting genome-wide expression profiles. Proc Natl Acad Sci U S A 102:15545-15550.

35. Lamparter D, Marbach D, Rueedi R, Kutalik Z, Bergmann S (2016): Fast and rigorous computation of gene and pathway scores from SNP-based summary statistics. PLoS Comput Biol 12:e1004714.

36. Supek F, Bosnjak M, Skunca N, Smuc T (2011): REVIGO summarizes and visualizes long lists of gene ontology terms. PLoS One 6:e21800.

37. Yeo M, Lee SK, Lee B, Ruiz EC, Pfaff SL, Gill GN (2005): Small CTD phosphatases function in silencing neuronal gene expression. Science 307:596-600.

38. Thiel G, Lietz M, Cramer M (1998): Biological activity and modular structure of RE-1-silencing transcription factor (REST), a repressor of neuronal genes. J Biol Chem 273:26891-26899.

39. Ertosun MG, Hapil FZ, Osman Nidai O (2016): E2F1 transcription factor and its impact on growth factor and cytokine signaling. Cytokine Growth Factor Rev 31:17-25. 
40. Phillips AC, Ernst MK, Bates S, Rice NR, Vousden KH (1999): E2F-1 potentiates cell death by blocking antiapoptotic signaling pathways. Mol Cell 4:771-781.

41. Hepper PG (2013): The developmental origins of laterality: Fetal handedness. Dev Psychobiol 55:588-595.

42. Rogers LJ, Andrew R (2002): Comparative Vertebrate Lateralization. Cambridge, UK: Cambridge University Press.

43. Rogers LJ, Vallortigara G (2015): When and why did brains break symmetry? Symmetry 7:2181-2194.

44. Chiandetti C, Galliussi J, Andrew RJ, Vallortigara G (2013): Early-light embryonic stimulation suggests a second route, via gene activation, to cerebral lateralization in vertebrates. Sci Rep 3:2701.

45. Corballis MC, Morgan MJ (1978): On the biological basis of human laterality: I. Evidence for a maturational left-right gradient. Behav Brain Sci 2:261-336.
46. Abu-Rustum RS, Ziade MF, Abu-Rustum SE (2013): Reference values for the right and left fetal choroid plexus at 11 to 13 weeks: An early sign of "developmental" laterality? J Ultrasound Med 32: 1623-1629.

47. Vallortigara G, Chiandetti C, Sovrano VA (2011): Brain asymmetry (animal). Wiley Interdiscip Rev Cogn Sci 2:146-157.

48. Govind CK (1992): Claw Asymmetry in Lobsters-Case-Study in Developmental Neuroethology. J Neurobiol 23:1423-1445.

49. Medland SE, Duffy DL, Wright MJ, Geffen GM, Hay DA, Levy F, et al. (2009): Genetic influences on handedness: Data from 25,732 Australian and Dutch twin families. Neuropsychologia 47:330-337.

50. Somers M, Ophoff RA, Aukes MF, Cantor RM, Boks MP, Dauwan M, et al. (2015): Linkage analysis in a Dutch population isolate shows no major gene for left-handedness or atypical language lateralization J Neurosci 35:8730-8736. 